\title{
Molecular-cytological analysis of common wheat lines with Triticum dicoccoides genetic material
}

\author{
Orlovskaya O.A. ${ }^{1 *}$, Leonova I.N. ${ }^{2}$, Solovey L.A. ${ }^{1}$, Dubovets N.I. ${ }^{1}$ \\ ${ }^{1}$ Institute of Genetics and Cytology NAS of Belarus, Minsk, Belarus \\ ${ }^{2}$ Institute of Cytology and Genetics, SB RAS, Novosibirsk, Russia \\ * email: O.Orlovskaya@igc.by
}

Wild emmer wheat ( $T$. dicoccoides Schweinf., AABB, $2 n=28)$ is characterized by resistant to many diseases and abiotic stressed factors, and is possessed high quality of the grain. The particular interest in this species as a donor of valuable traits is due to its close relationship with $T$. aestivum (both genomes of the wild emmer are homologous to the corresponding genomes of $T$. aestivum (AABBDD, $2 n=42)$ ), which facilitates the transfer of the target genes into the wheat. Hybrid wheat lines were obtained from crossing common wheat varieties with $T$. dicoccoides to enrich wheat gene pool with favorable genes. The aim of this study was to analyze the nature of alien introgression and their influence on the cytological stability of hybrid lines. C-banding, SSR- and SNP-analysis were used to assess the genomic composition of the lines. C-banding allowed to identify introgressions of $T$. dicoccoides genetic material in the common wheat genome in the form of whole chromosomes with a clear predominance of the Bgenome chromosomes $(6 \mathrm{~A}, 1 \mathrm{~B}, 2 \mathrm{~B}, 3 \mathrm{~B}, 5 \mathrm{~B})$. In only one line, alien chromatin was found in the form of chromosomal arms $\left(7 \mathrm{BS}^{\mathrm{d}}\right.$ and $\left.4 \mathrm{AL}^{\mathrm{d}}\right)$. Molecular methods showed the presence of a large number of $T$. dicoccoides fragments (9-12) in both genomes of the studied wheat line. The introgression of the B-genome genetic material of the wild species was carried out mainly in the form of whole arms or large fragments, while the insertions of the A-genome were less extended. The highest frequency of the introgressed fragments was observed in chromosomes 1A, 1B, 2B, 3B. Analysis of microsporogenesis revealed a high level of bivalent chromosome pairing (close to $100 \%$ ) and the meiotic index $(86,2-93,0 \%)$ in most lines. Introgression lines characterized by normal meiosis will be used for identification of genes determining resistance to biotic and abiotic factors and high grain quality in order to developed donor lines for breeding. Acknowledgements: The study was supported by BRFFR (projects B18R-028, B20R240) and RFBR (project No. 18-516-00001). 